\title{
Mimotope-P10s-PADRE Peptide Vaccine
}

National Cancer Institute

\section{Source}

National Cancer Institute. Mimotope-P10s-PADRE Peptide Vaccine. NCI Thesaurus. Code C123381.

A peptide-based vaccine containing a carbohydrate mimetic peptide (CMP) P10s fused to the pan HLA DR-binding epitope (PADRE) peptide, with immunomoadjuvant activity and potential antineoplastic activity. Upon injection of the mimotope-P10s-PADRE peptide vaccine, the P10s peptide, which mimics gang liosides and other tumor-associated carbohydrate antigens (TACA), both stimulates a cytotoxic T-lymphocyte (CTL) response towards cells expressing TACAs and induces the production of antibodies that are reactive with a broad set of TACAs. Additionally, the anti-TACA antibodies may interfere with cellular pathways involved in tumor cell survival and may induce antibody-dependent cellular cytotoxicity (ADCC) toward cells expressing TACAs. PADRE is a helper T-cell epitope that is able to increase the mag nitude and duration of the CT L response. 